\title{
Eph/ephrins-mediated thymocyte-thymic epithelial cell interactions control numerous processes of thymus biology
}

\author{
Javier Garcia-Ceca ${ }^{1}$, David Alfaro ${ }^{1}$, Sara Montero-Herradón ${ }^{1}$, Esther Tobajas ${ }^{1}$, \\ Juan José Muñoz ${ }^{2+}$ and Agustín G. Zapata ${ }^{1,2 *+}$ \\ ${ }^{1}$ Department of Cell Biology, Faculty of Biology, Complutense University, Madrid, Spain, ${ }^{2}$ Cytometry and Fluorescence \\ Microscopy Center, Complutense University, Madrid, Spain
}

OPEN ACCESS

Edited by:

Daniella Areas Mendes-da-Cruz, Fiocruz - Fundação Oswaldo Cruz,

Brazil

Reviewed by:

Yousuke Takahama,

University of Tokushima, Japan Andrew Freywald,

University of Saskatchewan, Canada

*Correspondence:

Agustín G. Zapata,

Department of Cell Biology, Faculty of Biology, Complutense University, José Antonio Novais 12, Madrid 28040, Spain

zapata@bio.ucm.es

† Juan José Muñoz and Agustín G. Zapata have contributed equally to this work.

Specialty section:

This article was submitted to Immunological Tolerance, a section of the journal Frontiers in Immunology

Received: 29 April 2015 Accepted: 15 June 2015

Published: 26 June 2015

Citation:

García-Ceca J, Alfaro D, Montero-Herradón S, Tobajas E, Muñoz JJ and Zapata AG (2015)

Eph/ephrins-mediated

thymocyte-thymic epithelial cell interactions control numerous processes of thymus biology.

Front. Immunol. 6:333. doi: 10.3389/fimmu.2015.00333
Numerous studies emphasize the relevance of thymocyte-thymic epithelial cell (TECs) interactions for the functional maturation of intrathymic T lymphocytes. The tyrosine kinase receptors, Ephs (erythropoietin-producing hepatocyte kinases) and their ligands, ephrins (Eph receptor interaction proteins), are molecules known to be involved in the regulation of numerous biological systems in which cell-to-cell interactions are particularly relevant. In the last years, we and other authors have demonstrated the importance of these molecules in the thymic functions and the T-cell development. In the present report, we review data on the effects of Ephs and ephrins in the functional maturation of both thymic epithelial microenvironment and thymocyte maturation as well as on their role in the lymphoid progenitor recruitment into the thymus.

Keywords: thymocytes, thymic epithelium, Eph, ephrin, thymic cell seeding

\section{Introduction}

The thymus is a lymphoid organ engaged in the production and homeostatic maintenance of functionally mature T cells, in which developing thymocytes interact sequentially with an epithelial network whose three-dimensional architecture is essential for the process. Thymocyte-thymic epithelial cell (TEC) interactions are, therefore, key for thymus functioning (1), and Eph and ephrins, two groups of molecules involved in these cell-to-cell contacts, have emerged as novel elements governing numerous thymic processes (2). Eph represent the largest group of receptor tyrosine kinases; they bind to surface ligands, ephrins and, according to their sequence homology and affinity for ephrins, are divided into EphA (10 members), which preferentially binds ephrins-A (6 members), ligands bound to the membrane through glycosylphosphatidylinositol, and EphB (6 members) that bind ephrins-B ( 3 members) that contains a transmembrane domain and a short cytoplasmic tail (3).

Eph/ephrins constitute an ubiquitous system due to the high number of members and their promiscuity, such that a single receptor can bind different ligands and vice versa, albeit with distinct affinities (4). Eph/ephrin-mediated interactions result in bidirectional signaling in the expressing cells, forward signals transmitted by Eph, and reverse in the ephrin-expressing cell (5), providing different cell responses depending on the multiple combinations and the direction of signaling (4). Eph/ephrins activate numerous signaling pathways that regulate cytoskeleton and cell adhesion but also gene transcription (6). 


\section{Eph and/or Ephrin are Expressed in the Thymus and Their Absence Results in Profound Thymic Hypocellularity}

Eph and ephrins, particularly those of the B group, are expressed widely in both thymocytes and TECs, frequently the same cell coexpressing the two types of molecules. They appear early in the thymic primordium (7-9) and a lack of these results in decreased numbers of both thymocytes (9-11) and TECs (12). The thymic hypocellularity of Eph/ephrin-deficient mice correlates with concomitant increased apoptosis affecting distinct thymocyte subsets (9-11). However, in all these Eph/ephrin-defective mice, it is difficult to establish conclusive correlations between thymic cellularity and thymocyte proliferation. Even in thymocyte-conditioned ephrin-B1/B2 thymuses, despite the evident reduced cellularity, there are increased proportions of proliferating DP thymocytes (11), suggesting some attempt to recover the thymic cell content (13).

Thymic epithelial cells (TECs) have been proposed to have a limited expanding capacity and the number of endodermal progenitors that organize the early thymic primordium could determine the final size of embryonic and adult thymus (14). Indeed, there is little information on the control of TEC survival and proliferation in general, and by the EphB group in particular. Developing thymuses of EphB2- and/or EphB3-deficient mice show increased TEC apoptosis largely affecting immature $\mathrm{EpCAM}^{+} \mathrm{MTS}^{+} 0^{+}$cells and $\mathrm{EpCAM}^{+} \mathrm{Ly} 51^{+}$cTECs, and in vitro activation of either EphB or ephrin-B signaling decreases the proportions of apoptotic WT TECs, whereas its disruption in RTOCs resulted in increased TEC death. Importantly, RTOCs established only with EphB-defective TECs yielded higher proportions of apoptotic cells than those observed when RTOCs were established with TECs and total thymocytes, suggesting that TEC survival is governed to a greater extent by Eph-ephrin-mediated thymocyte-TEC interactions (12).

On the other hand, decreased seeding of lymphoid progenitors, which periodically colonize the thymus, could also contribute to organ size and cellularity. Reduced lymphoid seeding into the thymus can be achieved by a reduction in the colonizing progenitor numbers and/or altered mechanisms of migration. Although, EphB2-deficient mice show decreased proportions of early BM hematopoietic progenitors compared to WT mice (15) their contribution to thymic seeding is a matter of discussion (16). On the contrary, BM cells expressing molecules known to be involved in thymus seeding (i.e., CCR7, CCR9, CXCR4, PSGL1) $(17,18)$ neither exhibit significant changes in EphB-deficient mice (15) nor in the numbers of fetal liver $\mathrm{CD} 45^{+} \mathrm{PIRA} / \mathrm{B}^{+}$precursors (unpublished data). Therefore, the lack of EphB affects the migratory capacity of progenitor cells rather than the proportions of colonizing cells.

In vivo and in vitro assays have demonstrated that the lack of EphB in either lymphoid progenitors or thymic stroma reduces thymic seeding in both fetal $(19,20)$ and adult mice $(15)$. In vitro colonization of WT FTOCs by EphB2 ${ }^{-1-}$ or EphB3 ${ }^{-1-}$, but not EphB2-LacZ, Lin ${ }^{-}$BM cells was significantly reduced compared to that shown by WT cells (19) and adult WT mice showed lower seeding into the thymus after in vivo injection of EphB-deficient
Lin $^{-}$BM cells than WT ones (15). This inability of progenitors to enter both fetal and adult thymus seems to be dependent, at least partially, on a direct role of Ephs in regulating cell migration, as previously reported for the cell migration of peripheral lymphocytes (21-23). In vitro migration of mutant progenitor BM cells was significantly reduced through fibronectin, laminin, or chemokine gradients, with a more severe reduction in EphB2-deficient cells than in EphB2-LacZ counterparts. Moreover, EphB2 stimulation by coated ephrin-B1Fc proteins inhibited laminin- and fibronectin-governing migration as well as CXCL12, CCL21, and CCL25-induced chemotaxis, but EphB2-LacZ cells did not exhibit reduced migration (19). This indicates that the extracellular domain of EphB promotes migration ligand independently while forward signaling promotes cell arrest.

In both experimental approaches, all tested BM progenitors, included WT ones, showed decreased migration into the EphBdeficient thymus, particularly the $\mathrm{EphB} 2^{-l-}$ one, which indicates the relevance of the thymic microenvironment in the process (15, 19). In fact, in both adult and fetal thymuses, decreased migration correlated with reduced production of ECM components, such as fibronectin and laminin, and chemokines (i.e., CXCL12, CCL21, CCL25) $(15,19)$. Furthermore, P-selectin involved in progenitor cell migration into the adult thymus (17), showed reduced expression on endothelial cells of both EphB2- and EphB3-deficient thymuses, but not of those of EphB2-LacZ cells, and decreased migration in $\mathrm{EphB} 2^{-1-}$ thymuses also correlated with reduced endothelial expression of ephrin-B1 and ephrin-B2, whereas in $\mathrm{EphB}^{-1-}$ thymuses, the reduction only affected ephrin-B1, reinforcing the idea that forward signals mediated by the pair EphB2/ephrin-B1 are particularly important for intrathymic lymphoid recruitment (15).

All these results, therefore, support a role for reduced thymic seeding in the thymic hypocellularity found in Eph/ephrin mutants. However, increased apoptosis of both thymocytes and TECs seems to be more relevant because, whereas thymuses deficient in both CCR7 and CCR9 with profoundly altered lymphoid colonization later recover normal thymocyte numbers (24), EphB2- and EphB3-deficient thymuses do not show that compensatory property; on the contrary, they increase their hypocellularity by increasing the death of DN and DP cells (9).

\section{Thymic Alterations Observed in Eph/Ephrin-deficient Mice Reflect the Relevance of Thymocyte-TEC Interactions}

Eph/ephrin deletion results in specific phenotypic alterations in both thymocytes and TECs. The lack of EphA4 results in a blockade of $\mathrm{T}$ cell maturation that results in a drop in DP cell proportions (10) and blockade of Eph/ephrin-A interactions in FTOCs treated with fusion proteins affects the maturation of immature $\mathrm{CD}^{-} \mathrm{CD}^{+}$thymocytes (2). In correlation, these thymuses show a profound collapse of the cortical epithelial network that significantly reduces the number of cell layers and their organization whereas immature $\mathrm{K}^{+} \mathrm{K}^{+}$TECs and areas devoid of epithelial cell marker expression increase. Apparently, the epithelial defects determine the lymphoid phenotype because mutant FTOCs grafted under the WT kidney capsule produce decreased 
proportions of DP thymocytes (10) while mutant thymocytes in a WT stroma do not reproduce these changes. On the contrary, in Eph/ephrin-B-deficient mice, alterations are very important in the epithelial component but less severe in the developing thymocytes $(9,11,25)$. EphB2- and EphB3-deficient thymuses exhibit minimal changes in the T-cell subset proportions, with an increased percentage of total DN cells and reduction of DN3 $\left(\mathrm{CD} 44^{-} \mathrm{CD} 25^{+}\right)$ cells (9). However, in this case, EphB acts cell-autonomously on T-cell differentiation as grafted EphB-deficient alymphoid fetal thymus lobes colonized by WT lymphoid progenitors exhibit normal T-cell differentiation (26), while chimeric thymuses generated with EphB2 $2^{-1-}$ and EphB2/B3 ${ }^{-1-}$ Lin $^{-}$BM cell progenitors injected into SCID mice showed a blockade of T-cell maturation at DN stage and chimeras established with $\mathrm{EphB} 3^{-1-}$ progenitor cells showed a partial blockade at this same point that resulted in low numbers of DP cells (27). Therefore, both EphB2 and EphB3 autonomously control thymocyte development at DN to DP transition. Both molecules are also necessary for the maturation of DP cells to SP thymocytes as demonstrated in reaggregates (RTOCs) formed with EphB2- or EphB3-deficient DP thymocytes and WT TECs (28). Eph expression on thymocytes is also important for thymocyte survival as in all these chimeric SCID mice there were increased proportions of apoptotic thymocytes, principally DP and SP CD4 ${ }^{+}$cells. Both thymocyte differentiation and survival seem to be dependent on Eph/ephrin-mediated thymocyte-TECs interaction and regulated by both forward and reverse signals, as SCID mice receiving EphB2-LacZ cells showed DP cells but did not produce SP thymocytes and did not show increased apoptosis. Therefore, although Eph forward signaling on thymocytes is necessary for thymocyte development, reverse signaling on interacting cells, presumably thymic epithelium, partially rescues DN cell progression to the DP cell compartment, and is important for cell survival (27). Accordingly, conditional deletion of ephrin-B1 and/or ephrin-B2 in TECs also affects the T-cell development and the lack of ephrin-B2 is presumably the most important, although ephrin-B1 also contributes, as double mutants show a more severe affectation (11). In addition, specific deletion of these ephrins in thymocytes results in a partial blockade of T-cell maturation at the DN3 stage $(11,29,30)$ and increased thymocyte apoptosis (11). The phenotype is similar in single and double mutants suggesting that both molecules have a cooperative rather than redundant role in thymocyte maturation (11). A similar phenotype, however, has not been found when EphB2- or EphB3-deficient thymocytes are developed in a WT stroma in a bone marrow transplantation experiment into SCID mice (26).

Eph and ephrin signaling also affect thymic epithelium development and organization as in both EphB- and ephrin-Bmutant mice there is a profound transformation of thymic epithelium that exhibits altered TEC phenotypes (i.e., immature $\mathrm{K}^{+} \mathrm{K}^{+}{\mathrm{MTS} 10^{+}}^{+}$medullary epithelial cells (mTECs), cortical $\mathrm{K}^{-} \mathrm{K}^{-}{ }^{-} \mathrm{MTS}_{20}{ }^{+}$cells and $\mathrm{K}^{+}{ }^{+} 8^{+}$cells) and altered $3 \mathrm{D}$ organization. This change provokes a $2 \mathrm{D}$ structure that results in increased epithelial cysts, collapsed epithelium, and large areas devoid of epithelial cell markers $(11,25,31)$. These latter areas, of unknown significance, also exist in WT thymuses and in other mice with defects in molecules, such as Foxn1, Kremen 1, or Stat3, involved in TEC maturation (32-34), but are specially developed in EphB-deficient thymuses. They contain thymocytes and blood vessels, frequently surrounded by enlarged sheaths of connective tissue, and are different in cortex and medulla: the former ones contain thymocytes and some sheathed blood vessels, whereas in the medulla mTECs delimit areas with enlarged blood vessels, increased numbers of ER-TR7 ${ }^{+}$fibroblasts, components of the ECM (collagen IV, fibronectin, and laminin) (35), dendritic cells (36), and thymocytes in some areas (37-39).

Cortical areas devoid of epithelium have been described by others (37-39), receiving the name of epithelial-free areas (EFAs). EFAs are MHC class-II negative, little vascularized areas that contain abundant thymocytes frequently in division (39) reported as accumulations of DP thymocytes that do not undergo positive selection and will die subsequently by apoptosis (40). On the contrary, medullary epithelium-free areas that express several connective tissue markers could have a mesenchymal condition (35) and represent areas in which Eph-deficient TECs have undergone an epithelial-mesenchymal transition, losing their epithelial cell markers and acquiring a mesenchymal nature. In Eph mutant mice, EFAs could arise as a consequence of impeded intermingling and mutual exclusion of thymocytes and TECs caused by the lack of Eph-ephrin signaling as known in other systems (41).

Presumably, TEC maturation is autonomously governed by EphB2 and EphB3 expressed on TECs, as some of the phenotypical alterations found in EphB2- or EphB3-deficient mice can be reproduced in grafted mutant lobes colonized by WT host thymocytes. However, EphB expressed on thymocytes can also play a non-autonomous role since the epithelial phenotype of these grafted mutant lobes was not exactly the same as that found in EphB-deficient thymuses (26), and chimeric SCID thymuses receiving EphB-deficient thymocytes showed altered histological organization (27).

Selective deletion of ephrin-B1 and/or ephrin-B2 genes in thymocytes or TECs permits to determine the relevance of Eph/ephrin signaling in distinct thymic components (11). In all ephrin-B-deficient mice, but particularly in the double mutants with ephrin-B1 and ephrin-B2 deleted in TECs, the thymuses are small, with scarcely developed cortex and medulla, high numbers of $\mathrm{K}^{+} \mathrm{K}^{+}$cells, and numerous epithelial cysts. Ephrin-B2 deletion in TECs causes altered distribution of $\mathrm{Ly}_{51}{ }^{+}$cortical (c) TEC subsets defined as Ly51 ${ }^{\text {hi }}$ cells that express DLL4, and would constitute the cortical niche of DN thymocytes and Ly51 $1^{\text {lo }}$ cTECs that would represent that of DP cells. Thus, ephrin-B1 deletion in TECs but, also, the lack of ephrin-B1 and B2 in thymocytes induce a cortex in which rounded groups of cTECs express Ly51 homogeneously, suggesting that ephrin-B1 and B2 expressed in both thymocytes and TECs cooperate in regulating the differentiation and distribution of cortical niches (11). Furthermore, ephrin-B deletion from both thymocytes and TECs affects medulla organization. The medulla of thymuses bearing a deletion of ephrin-B1 or B2 in thymocytes, or those without ephrin-B1 or ephrin-B1/B2 in TECs, shows increased numbers of large monolayered epithelial cysts formed largely by immature $\mathrm{K}^{+} \mathrm{K}^{+}$, sometimes $\mathrm{MTS}_{20}{ }^{+}$, TECs, but rarely containing mature $\mathrm{UEA} 1^{+}$ or $\mathrm{MTS}^{+} 0^{+}$cells that would represent an arrest of medulla development at an immature stage. They also contain numerous UEA $1^{\text {hi }}$ cells that form small cysts surrounded by a thin rim of 
$\mathrm{UEA}^{\mathrm{lo} /-} \mathrm{MTS}^{+}{ }^{+}$cells that would represent a certain blockade of medulla organization at a late stage of development, in which medullary TEC subsets UEA $1^{\text {hi }} \mathrm{MTS}^{-} 0^{-}$and UEA $1^{\text {lo }} \mathrm{MTS} 0^{+}$ develop but $\mathrm{MTS}^{+} 0^{+}$cell expansion and 3D organization are affected (31).

Interestingly, these studies support that ephrin-B1 and ephrinB2 deletion in TECs result in different TEC phenotypes similar to those generated by ephrin-B1 or ephrin-B2 deletion in thymocytes. This, once again, indicates that Eph/ephrinmediated thymocyte-TEC interactions are also important for TEC development and arrangement. Although, these molecules also mediate homotypic interactions (thymocyte-thymocyte; TEC-TEC), presumably their involvement in thymocyte-TEC interactions is more important to explain their role in the thymus. However, there are no complementary phenotypes when the effects of ephrin deletion in TECs are compared with those observed in thymuses with EphB-deficient epithelium, or when Eph mutant or ephrin mutant phenotypes are compared. This indicates that in the thymus, as in other systems (4), the final balance of forward and reverse signals in thymocytes and/or TECs would be more relevant than the mere presence/absence of certain Eph or ephrins. Besides, other factors must contribute to the complexity of the system as phenotypes in different mutant models are more severe in mice with C57/Bl6-CD1 mixed background than in the non-inbreed strain C57/Bl6 (10, 11).

In vitro experiments also clearly support the relevance of Eph/ephrin-mediated thymocyte-TEC interactions in thymus biology: ephrin-B1Fc proteins added to RTOCs, formed by fetal TECs and DP thymocytes, disorganize the 3D thymic epithelial network, prevent thymocyte-TEC association, and alter TCR $\alpha \beta$ signaling (28). Numbers and timing of the establishment of cell conjugates also change when they are established with EphBdeficient DP thymocytes (27).

On the other hand, proper T-cell maturation occurs thanks to the movement of developing thymocytes throughout the thymic parenchyma, facilitating their interactions with distinct niches favoring the necessary thymocyte-TEC crosstalk (42). Analysis by confocal microscopy of the positioning of

\section{References}

1. Boyd RL, Tucek CL, Godfrey DI, Izon DJ, Wilson TJ, Davidson NJ, et al. The thymic microenvironment. Immunol Today (1993) 14(9):445-59. doi:10.1016/ 0167-5699(93)90248-J

2. Munoz JJ, Cejalvo T, Alonso-Colmenar LM, Alfaro D, Garcia-Ceca J, Zapata A. Eph/ephrin-mediated interactions in the thymus. Neuroimmunomodulation (2011) 18(5):271-80. doi:10.1159/000329490

3. Gale NW, Holland SJ, Valenzuela DM, Flenniken A, Pan L, Ryan TE, et al. Eph receptors and ligands comprise two major specificity subclasses and are reciprocally compartmentalized during embryogenesis. Neuron (1996) 17(1):9-19. doi:10.1016/S0896-6273(00)80276-7

4. Pasquale EB. Eph-ephrin bidirectional signaling in physiology and disease. Cell (2008) 133(1):38-52. doi:10.1016/j.cell.2008.03.011

5. Knoll B, Drescher U. Ephrin-as as receptors in topographic projections. Trends Neurosci (2002) 25(3):145-9. doi:10.1016/S0166-2236(00)02093-2

6. Poliakov A, Cotrina M, Wilkinson DG. Diverse roles of eph receptors and ephrins in the regulation of cell migration and tissue assembly. Dev Cell (2004) 7(4):465-80. doi:10.1016/j.devcel.2004.09.006
EphB2-deficient or WT $\mathrm{Lin}^{-} \mathrm{BM}$ progenitors in reconstituted FTOCs demonstrated that higher numbers of WT cells reached the central area of WT lobes than of EphB2-deficient cells (19). Furthermore, EphB2 $2^{-l-}$ total thymocytes migrate less efficiently through laminin or fibronectin or in response to CXCL12, CCL21, or CCL25, than WT cells. More importantly, when forward EphB2 signals were activated by ephrin$\mathrm{B} 1 \mathrm{Fc}$ protein treatment, there was a significant reduction in the migration of all EphB2 ${ }^{-1-}$, but not EphB2-LacZ, thymocyte subsets. Therefore, together with chemokines and ECM molecules, the migration of developing thymocytes throughout the thymic stroma could be promoted by inactivated EphB2 receptors, and negatively modulated by EphB2/ephrin-B interactions (19).

Remarkably, these profound phenotypic alterations observed in mice deficient in distinct Eph or ephrins do not correlate with immune deficiencies and/or pathological processes. EphA4 ${ }^{-1-}$ thymuses (10) and those with deleted ephrin-B1 and/or ephrin-B2 in TECs (11) show decreased proportions of both DP TCR $\alpha \beta^{\text {hi }}$ cells and CD69+ ${ }^{+}$cells that could reflect an inefficient TCR $\alpha \beta$ selection. However, peripheral lymphoid organs of both EphA4 ${ }^{-1-}$ mice (10) and EphB-deficient mice (9) show decreased numbers of total $\mathrm{T}$ cells, but not significant changes in the proportions of distinct T-cell subsets. In addition, there are no changes in the central and peripheral TCR $\alpha \beta$ repertoire expressed on $\mathrm{CD}^{+}{ }^{+} \mathrm{T}$ cells of EphB2- and/or EphB3-mutant mice, except for an increased proportion of $\mathrm{V} \beta 3^{+} \mathrm{CD} 4^{+}$cells in both thymus and lymph nodes of the three mutants (43).

In summary, Eph and ephrins are molecules that through mediating thymocyte-TEC interactions are involved in numerous processes occurring into the thymus, including cell migration into and through thymus, T-cell differentiation, and TEC maturation.

\section{Acknowledgments}

This work was supported by grants RD06/0010/0003 and RD12/0019/0007, from the Spanish Health Ministry; BFU201018250 and BFU2013-41112-R, from the Spanish Ministry of Economy and Competitiveness.

7. Munoz JJ, Alonso CL, Sacedon R, Crompton T, Vicente A, Jimenez E, et al. Expression and function of the Eph A receptors and their ligands ephrins A in the rat thymus. J Immunol (2002) 169(1):177-84. doi:10.4049/jimmunol. 169.1.177

8. Vergara-Silva A, Schaefer KL, Berg LJ. Compartmentalized Eph receptor and ephrin expression in the thymus. Mech Dev (2002) 119(Suppl 1):S225-9. doi:10. 1016/S0925-4773(03)00121-7

9. Alfaro D, Munoz JJ, Garcia-Ceca J, Cejalvo T, Jimenez E, Zapata A. Alterations in the thymocyte phenotype of EphB-deficient mice largely affect the double negative cell compartment. Immunology (2008) 125(1):131-43. doi:10.1111/j. 1365-2567.2008.02828.x

10. Munoz JJ, Alfaro D, Garcia-Ceca J, Alonso CL, Jimenez E, Zapata A. Thymic alterations in EphA4-deficient mice. J Immunol (2006) 177(2):804-13. doi:10. 4049/jimmunol.177.2.804

11. Cejalvo T, Munoz JJ, Tobajas E, Fanlo L, Alfaro D, Garcia-Ceca J, et al. EphrinB-dependent thymic epithelial cell-thymocyte interactions are necessary for correct $\mathrm{T}$ cell differentiation and thymus histology organization: relevance for thymic cortex development. J Immunol (2013) 190(6):2670-81. doi:10.4049/ jimmunol.1201931 
12. Garcia-Ceca J, Alfaro D, Montero-Herradon S, Zapata AG. Eph/ephrinB signalling is involved in the survival of thymic epithelial cells. Immunol Cell Biol (2013) 91(2):130-8. doi:10.1038/icb.2012.59

13. Yu G, Mao J, Wu Y, Luo H, Wu J. Ephrin-B1 is critical in T-cell development. J Biol Chem (2006) 281(15):10222-9. doi:10.1074/jbc.M510320200

14. Jenkinson WE, Bacon A, White AJ, Anderson G, Jenkinson EJ. An epithelial progenitor pool regulates thymus growth. J Immunol (2008) 181(9):6101-8. doi:10.4049/jimmunol.181.9.6101

15. Alfaro D, Garcia-Ceca J, Farias-de-Oliveira DA, Terra-Granado E, MonteroHerradon S, Cotta-de-Almeida V, et al. EphB2 and EphB3 play an important role in the lymphoid seeding of murine adult thymus. J Leukoc Biol (2015). doi:10.1189/jlb.1HI1114-568R

16. Zhang SL, Bhandoola A. Trafficking to the thymus. Curr Top Microbiol Immunol (2014) 373:87-111. doi:10.1007/82_2013_324

17. Gossens K, Naus S, Corbel SY, Lin S, Rossi FM, Kast J, et al. Thymic progenitor homing and lymphocyte homeostasis are linked via S1P-controlled expression of thymic P-selectin/CCL25. J Exp Med (2009) 206(4):761-78. doi:10.1084/jem. 20082502

18. Zlotoff DA, Sambandam A, Logan TD, Bell JJ, Schwarz BA, Bhandoola A. CCR7 and CCR9 together recruit hematopoietic progenitors to the adult thymus. Blood (2010) 115(10):1897-905. doi:10.1182/blood-2009-08-237784

19. Stimamiglio MA, Jimenez E, Silva-Barbosa SD, Alfaro D, Garcia-Ceca JJ, Munoz JJ, et al. EphB2-mediated interactions are essential for proper migration of T cell progenitors during fetal thymus colonization. J Leukoc Biol (2010) 88(3):483-94. doi:10.1189/jlb.0210079

20. Mendes-da-Cruz DA, Stimamiglio MA, Munoz JJ, Alfaro D, Terra-Granado E, Garcia-Ceca J, et al. Developing T-cell migration: role of semaphorins and ephrins. FASEB J (2012) 26(11):4390-9. doi:10.1096/fj.11-202952

21. Sharfe N, Freywald A, Toro A, Dadi H, Roifman C. Ephrin stimulation modulates T cell chemotaxis. Eur J Immunol (2002) 32(12):3745-55. doi:10.1002/ 1521-4141(200212)32:12<3745:AID-IMMU3745>3.0.CO;2-M

22. Aasheim HC, Delabie J, Finne EF. Ephrin-A1 binding to CD4+ T lymphocytes stimulates migration and induces tyrosine phosphorylation of PYK2. Blood (2005) 105(7):2869-76. doi:10.1182/blood-2004-08-2981

23. Trinidad EM, Ballesteros M, Zuloaga J, Zapata A, Alonso-Colmenar LM. An impaired transendothelial migration potential of chronic lymphocytic leukemia (CLL) cells can be linked to ephrin-A4 expression. Blood (2009) 114(24):5081-90. doi:10.1182/blood-2009-03-210617

24. Liu C, Saito F, Liu Z, Lei Y, Uehara S, Love P, et al. Coordination between CCR7- and CCR9-mediated chemokine signals in prevascular fetal thymus colonization. Blood (2006) 108(8):2531-9. doi:10.1182/blood-2006-05-024190

25. Garcia-Ceca J, Jimenez E, Alfaro D, Cejalvo T, Chumley MJ, Henkemeyer M, et al. On the role of Eph signalling in thymus histogenesis; EphB2/B3 and the organizing of the thymic epithelial network. Int J Dev Biol (2009) 53(7):971-82. doi: $10.1387 / \mathrm{ijdb} .082702$ jg

26. Garcia-Ceca J, Jimenez E, Alfaro D, Cejalvo T, Munoz JJ, Zapata AG. Cell-autonomous role of EphB2 and EphB3 receptors in the thymic epithelial cell organization. Eur J Immunol (2009) 39(10):2916-24. doi:10.1002/eji. 200939437

27. Alfaro D, Munoz JJ, Garcia-Ceca J, Cejalvo T, Jimenez E, Zapata AG. The Eph/ephrinB signal balance determines the pattern of T-cell maturation in the thymus. Immunol Cell Biol (2011) 89(8):844-52. doi:10.1038/icb.2010.172

28. Alfaro D, Garcia-Ceca JJ, Cejalvo T, Jimenez E, Jenkinson EJ, Anderson $\mathrm{G}$, et al. EphrinB1-EphB signaling regulates thymocyte-epithelium interactions involved in functional T cell development. Eur J Immunol (2007) 37(9):2596-605. doi:10.1002/eji.200737097
29. Jin W, Qi S, Luo H. The effect of conditional EFNB1 deletion in the T cell compartment on T cell development and function. BMC Immunol (2011) 12:68. doi:10.1186/1471-2172-12-68

30. Luo H, Charpentier T, Wang X, Qi S, Han B, Wu T, et al. Efnb1 and Efnb2 proteins regulate thymocyte development, peripheral $\mathrm{T}$ cell differentiation, and antiviral immune responses and are essential for interleukin-6 (IL-6) signaling. J Biol Chem (2011) 286(48):41135-52. doi:10.1074/jbc.M111.302596

31. Cejalvo T, Munoz JJ, Tobajas E, Alfaro D, Garcia-Ceca J, Zapata A. Conditioned deletion of ephrinB1 and/or ephrinB2 in either thymocytes or thymic epithelial cells alters the organization of thymic medulla and favors the appearance of thymic epithelial cysts. Histochem Cell Biol (2015) 143(5):517-29. doi:10.1007/ s00418-014-1296-9

32. Sano S, Takahama Y, Sugawara T, Kosaka H, Itami S, Yoshikawa K, et al. Stat3 in thymic epithelial cells is essential for postnatal maintenance of thymic architecture and thymocyte survival. Immunity (2001) 15(2):261-73. doi:10. 1016/S1074-7613(01)00180-7

33. Su DM, Navarre S, Oh WJ, Condie BG, Manley NR. A domain of Foxn1 required for crosstalk-dependent thymic epithelial cell differentiation. Nat Immunol (2003) 4(11):1128-35. doi:10.1038/ni983

34. Osada M, Ito E, Fermin HA, Vazquez-Cintron E, Venkatesh T, Friedel RH, et al. The Wnt signaling antagonist Kremen 1 is required for development of thymic architecture. Clin Dev Immunol (2006) 13(2-4):299-319. doi:10.1080/ 17402520600935097

35. Clarke AG, Gil AL, Kendall MD. The effects of pregnancy on the mouse thymic epithelium. Cell Tissue Res (1994) 275(2):309-18. doi:10.1007/BF00319429

36. Sawanobori Y, Ueta H, Dijkstra CD, Park CG, Satou M, Kitazawa Y, et al. Three distinct subsets of thymic epithelial cells in rats and mice defined by novel antibodies. PLoS One (2014) 9(10):e109995. doi:10.1371/journal.pone.0109995

37. von Gaudecker B. The development of the human thymus microenvironment Curr Top Pathol (1986) 75:1-41.

38. Godfrey DI, Izon DJ, Tucek CL, Wilson TJ, Boyd RL. The phenotypic heterogeneity of mouse thymic stromal cells. Immunology (1990) 70(1):66-74.

39. Bruijntjes JP, Kuper CF, Robinson JE, Schuurman HJ. Epithelium-free area in the thymic cortex of rats. Dev Immunol (1993) 3(2):113-22. doi:10.1155/1993/ 90759

40. Boyd RL, Hugo P. Towards an integrated view of thymopoiesis. Immunol Today (1991) 12(2):71-9. doi:10.1016/0167-5699(91)90161-L

41. Wilkinson DG. Regulation of cell differentiation by Eph receptor and ephrin signaling. Cell Adh Migr (2014) 8(4):339-48. doi:10.4161/19336918.2014.970007

42. Anderson G, Takahama Y. Thymic epithelial cells: working class heroes for $\mathrm{T}$ cell development and repertoire selection. Trends Immunol (2012) 33(6):256-63. doi:10.1016/j.it.2012.03.005

43. Munoz JJ, Alfaro D, Garcia-Ceca J, Cejalvo T, Stimamiglio MA, Jimenez E, et al Eph and ephrin: key molecules for the organization and function of the thymus gland. Immunología (2009) 28(1):19-31. doi:10.1111/j.1749-6632.2008.03965.x

Conflict of Interest Statement: The authors declare that the research was conducted in the absence of any commercial or financial relationships that could be construed as a potential conflict of interest.

Copyright (C) 2015 García-Ceca, Alfaro, Montero-Herradón, Tobajas, Muñoz and Zapata. This is an open-access article distributed under the terms of the Creative Commons Attribution License (CC BY). The use, distribution or reproduction in other forums is permitted, provided the original author(s) or licensor are credited and that the original publication in this journal is cited, in accordance with accepted academic practice. No use, distribution or reproduction is permitted which does not comply with these terms. 